\title{
Similarity Metrics for Groupwise Non-rigid Registration
}

\author{
Kanwal K. Bhatia ${ }^{1}$, Jo Hajnal ${ }^{2}$, Alexander Hammers ${ }^{2,3}$, and Daniel Rueckert ${ }^{1}$ \\ ${ }^{1}$ Visual Information Processing, Department of Computing, Imperial College London \\ ${ }^{2}$ Imaging Sciences Department, MRC Clinical Sciences Centre, Imperial College \\ London, Hammersmith Hospital \\ ${ }^{3}$ Division of Neuroscience, Faculty of Medicine, Imperial College London, \\ Hammersmith Hospital
}

\begin{abstract}
The use of groupwise registration techniques for average atlas construction has been a growing area of research in recent years. One particularly challenging component of groupwise registration is finding scalable and effective groupwise similarity metrics; these do not always extend easily from pairwise metrics. This paper investigates possible choices of similarity metrics and additionally proposes a novel metric based on Normalised Mutual Information. The described groupwise metrics are quantitatively evaluated on simulated and 3D MR datasets, and their performance compared to equivalent pairwise registration.
\end{abstract}

\section{Introduction}

There has been growing interest in unbiased atlas construction through the use of groupwise registration [1] [2] [3] [4] 6]. Techniques to do this often do not require the choice of any reference image, and the atlas obtained is therefore not biased by such a choice. The groupwise registration methods developed vary in terms of deformation model, similarity metric and averaging principle used, however, little comparison of such methods has been made. While the deformation models generally follow naturally from comparable pairwise methods, the extension of metrics representing the similarity between two images to the similarity between all subjects in a group, is a more challenging task. Similarity metrics should scale at most linearly with increasing numbers of subjects while still remaining effective at driving registration.

Entropy-based similarity metrics are frequently used in pairwise non-rigid registration. Their evaluation typically requires the evaluation of probability density functions, for example, through the use of histograms. Extending these metrics to multiple images requires the evaluation of multidimensional PDFs. For example, extending Normalised Mutual Information (NMI) [1] 7] to $n$ images gives:

$$
S=\frac{1}{H\left(I_{1}, I_{2} \ldots I_{n}\right)} \sum_{i=1}^{n} H\left(I_{i}\right)
$$

where $H\left(I_{i}\right)$ represents the marginal entropy of image $i$ and $H\left(I_{1}, I_{2}, \ldots I_{n}\right)$ represents the joint entropy of all the images. Evaluating $n$-dimensional NMI,

N. Ayache, S. Ourselin, A. Maeder (Eds.): MICCAI 2007, Part II, LNCS 4792, pp. 544 552, 2007.

(C) Springer-Verlag Berlin Heidelberg 2007 
therefore requires an $n$-dimensional histogram. Apart from the exponentially increasing memory requirements, this also leads to increasing sparsity as the histogram size becomes very much larger than the number of samples contained. For example, 10 images of size $256 \times 256 \times 256$ with 64 intensity bins, gives a histogram size of $64^{10}=2^{60}$, but only $256^{3}=2^{24}$ samples. The evaluation of such sparse histograms is computationally inefficient and so this method is infeasible for large numbers of subjects.

Previous attempts to extend joint entropy-based metrics have been proposed by [1] and [3]. In [1], a histogram is created with the same dimensionality as the number of subjects. Evaluating this histogram requires the use of a parallel cluster of processors. 3 proposed the selection of one arbitrary image of the population to act as an intensity reference. A joint histogram is built with pair of intensities, comprising the voxel intensity in the reference and the corresponding intensity in each subject. A problem with this method is that with an increasingly large number of intensity pairs, for example when using a large number of 3D images, the effect that each pair has on the overall evaluation of the histogram is greatly reduced.

In this paper we propose an alternate extension to NMI. Furthermore, we evaluate different similarity metrics for groupwise non-rigid registration and evaluate their performance on simulated as well as real 3D MR data. Additionally, we assess groupwise registration in comparison to iterative pairwise techniques for average atlas construction.

\section{Methods}

\subsection{Groupwise Registration}

The groupwise registration algorithm used in this paper is based on 3. which uses a free-form deformation model based on B-splines. The deformations of corresponding points are constrained to sum to zero in order to ensure that the resulting atlas represents the average shape of the population.

\subsection{Similarity Metrics}

Similarity metrics that can be used efficiently within the above groupwise registration framework above are described in the following sections. All the metrics described scale linearly with increasing numbers of images in the population.

Voxel Intensity-Based Metrics. Previous work on groupwise registration [2] 8] has used voxelwise differences from a reference intensity (for example the mean voxel intensity or the intensity of a selected reference image). One such measure when a reference image is not selected, is the sample variance of a population, which represents the difference from the current mean intensity $\bar{I}(\mathbf{x})$ for each voxel $\mathbf{x}$ in image domain $\Omega$ :

$$
S_{S V}=\sum_{i=1}^{n} \sum_{\mathbf{x} \in \Omega} \frac{(I(\mathbf{x})-\bar{I}(\mathbf{x}))^{2}}{n_{\Omega}}
$$

The mean intensity is updated at every iteration of the registration process. 
Entropy-Based Metrics. In pairwise registration, NMI has been shown to be an effective similarity measure [7, and it would be desirable to extend this to groupwise regisrtation. We propose a novel measure whereby separate histograms are constructed using each individual image and an average intensity image of the population. The similarity is the sum of the individual NMI values:

$$
S_{A N M I}=\sum_{i=1}^{n}\left(\frac{H(\bar{I})+H\left(I_{i}\right)}{H\left(\bar{I}, I_{i}\right)}\right)
$$

The reference image, $\bar{I}$, is the current voxel-wise mean intensity of the group of images and is updated at every iteration.

Label Consistency (LC). The use of multiple images can potentially cause issues due to the increased dimensionality and increased variation in intensities. This motivates the use of segmentation-based metrics for multi-subject similarity. If hard segmentations of every subject in the population are available, the overlap between these structures could be used to align the images. The label consistency is one such metric:

$$
S_{L C}=\sum_{i=1}^{n} \frac{N\left(I_{i} \cap I_{\text {ref }}\right)}{N\left(I_{i} \cup I_{\text {ref }}\right)}
$$

where $N\left(I_{i} \cap I_{r e f}\right)$ represents the number of voxels in both image $I_{i}$ and the reference image having the same label and $\left(I_{i} \cup I_{r e f}\right)$ is the total of the number of voxels labelled in $I_{i}$ and the reference. One choice for the reference image $I_{r e f}$ is the maximum probability estimate [9, created by assigning to each voxel the class representing the mode of the group (i.e. the most commonly occuring class at that voxel). This reference is recalculated at every iteration.

Kullback Leibler Divergence (KL). An issue with using hard segmentations is that labelled regions may span many different structures. There is no way for the registration to tell how well individual structures are aligned within the same labelled area. Probabilistic segmentations are likely to offer greater sensitivity. These can be obtained via, for example, the Expectation-Maximisation (EM) algorithm [10]. The Kullback-Leibler divergence [1] is an additive measure which measures the distance between PDFs, and has previously been used for groupwise registration in [6]. For images segmented into $K$ tissue classes:

$$
S_{K L}=\sum_{i=1}^{n} \sum_{\mathbf{x} \in \Omega} \sum_{k}^{K} p_{i, \mathbf{x}, k} \log \frac{p_{i, \mathbf{x}, k}}{p_{r e f, \mathbf{x}, k}}
$$

where $p_{i, \mathbf{x}, k}$ is the probability of voxel location $\mathbf{x}$ in image $i$ being classified as tissue class $k$, and the reference probability is taken as the mean of the probabilities at that voxel:

$$
p_{r e f, \mathbf{x}, k}=\frac{\sum_{i} p_{i, \mathbf{x}, k}}{n}
$$

Once again, the reference image has to be updated at each iteration of the registration. 
Iterative Pairwise Methods for Average Atlas Construction. Average atlas construction need not be performed in a purely groupwise fashion. Algorithms for the application of average transformations from pairwise registrations have also been developed [12, [13]. The method considered in this paper is directly comparable to the groupwise method in that it uses the same free-form deformation model based on B-splines [7. NMI is used as a similarity metric to pairwise register all subjects to a chosen reference subject. The inverse of the mean of these deformations is then applied to transform each image to the average shape [13. This process can be repeated by iteratively re-registering to the current average image as in 12 .

\subsection{Criteria for the Evaluation of Similarity Metrics}

To analyse the performance of the metrics described, two items need to be considered:

1. Accuracy: how well the registration recovers the average shape of the population for each image.

2. Consistency: how well-aligned the subjects in the group are with each other.

Accuracy. The accuracy of registration can be assessed if the average shape of the population or the transformations to the average shape are known. If tissue classes are available, the Dice similarity metric can be used:

$$
D=\frac{2 \times N\left(I \cap I_{\text {ref }}\right)}{N\left(I \cup I_{\text {ref }}\right)}
$$

which is twice the ratio of the number of voxels correctly labelled to the total number of voxels with that label in both the reference and image under consideration. If the transformation is known, the average absolute displacement error for each voxel, x, can be computed:

$$
\text { Error }=\sum_{\mathbf{x} \in \Omega} \frac{\left|d_{\text {simulated }}(\mathbf{x})-d_{\text {recovered }}(\mathbf{x})\right|}{n_{\Omega}}
$$

Consistency. How well-registered the subjects are with each other is determined using two measures: entropy and accumulated overlap of structures. The more well-aligned a population is, the sharper the resulting final atlas (a mean of the intensities of the individual transformed images) should be. However, it may not be easy to distinguish between atlases by visual inspection alone. The entropy, $\mathrm{H}(\mathrm{A})$ of each atlas is therefore computed:

$$
H(A)=-\sum_{\mathbf{x}} p(A(\mathbf{x})) \log p(A(\mathbf{x}))
$$

where $p(A(\mathbf{x}))$ is the probability of the intensity of voxel $\mathbf{x}$. As the atlas gets sharper, its entropy should decrease. Groupwise accumulated overlaps based on 
fuzzy set theory, developed by Crum et al. [14], have also been used to assess the registration:

$$
\text { Overlap }=\frac{\sum_{\text {pairs }} \sum_{\text {labels }} \sum_{\text {voxels }} \min \left(I_{1}, I_{2}\right)}{\sum_{\text {pairs }} \sum_{\text {labels }} \sum_{\text {voxels }} \max \left(I_{1}, I_{2}\right)}
$$

where $I_{1}$ and $I_{2}$ are the binary segmentation values in a given pair of images for a given voxel and label.

\section{Results}

A set of 100 deformation fields was created such that the total deformation of the set was equal to zero. The inverses of these deformations (calculated using a numerical scheme [15) were applied to a slice of the MNI Brainweb image to create a population of 100 subjects, whose average shape is the original, undeformed slice. In addition, the MNI Brainweb image has probabilistic and ground truth (hard) segmentations of white matter (WM), grey matter (GM), cerebrospinal fluid (CSF) and background (BG) classes. These were also transformed to the space of each individual image for use in segmentation-based registration techniques and in the evaluation of the results. Groupwise registration of the population using each of the similarity metrics was performed: sample variance (SV), label consistency (LC), Kullback-Leibler divergence (KL) and normalised mutual information with the average image (ANMI). In addition, an average atlas of the population was created by using the mean transformation of pairwise registrations to a chosen subject $(\mathrm{P}(\mathrm{i}=1))$. This atlas was updated by reregistering each subject to the current atlas, and using the new transformations to update the atlas, up to four times $(\mathrm{P}(\mathrm{i}=2), \mathrm{P}(\mathrm{i}=3), \mathrm{P}(\mathrm{i}=4))$. The final atlases are shown in Figure 1 .
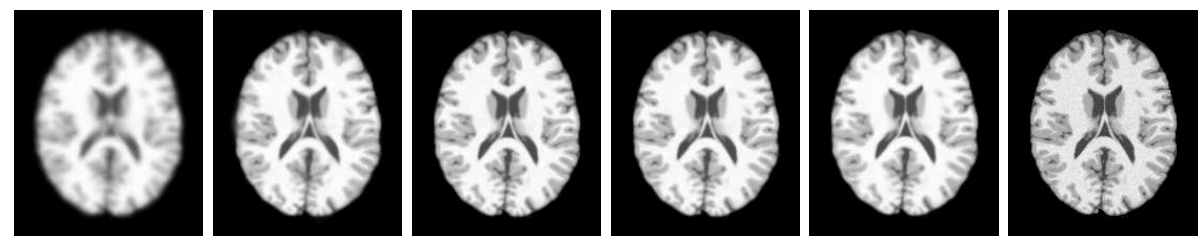

Fig. 1. Far left: atlas of original population. L-R: average shape atlases using $\mathrm{P}(\mathrm{i}=1)$, KL, ANMI and SV registration methods. Far right: MNI Brainweb image.

\subsection{Accuracy}

The accuracy of the registrations are determined by how well the registrations recover the original MNI Brainweb image. The overlaps between tissue classes obtained through registration and the actual segmentations are shown in Figure 2(a), while the errors in the obtained deformation fields are shown in 2(b). 
It can be seen that best performing groupwise metrics are the KL and ANMI metrics, which provide results close to those obtained when using four iterations of the pairwise registration to the average shape. As expected, the label consistency metric performs poorly in comparison. For this metric to work well, more segmentations of smaller structures would be needed.
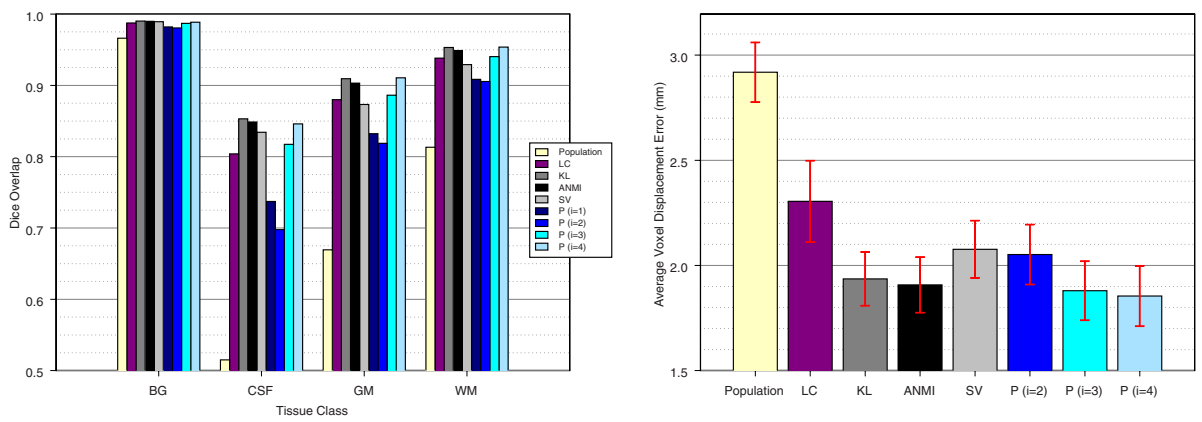

Fig. 2. Dice overlaps and deformation errors indicating accuracy of registration

\subsection{Consistency}

The entropies of the final atlases and the accumulated overlaps are shown in Figure 3 It should be noted that the accumulated overlap measure implicitly weights the effect of structures according to their area. In contrast, entropy weights all voxels equally and hence there may not be a direct relationship between the two. In Figure 3(b), the results shown do not include the alignment of the very large background class. Once again, the best methods are four iterations of the pairwise registration and the KL and ANMI metrics for groupwise registration. However, the entropy shows that the iterative pairwise method does not guarantee increasing consistency with each iteration.
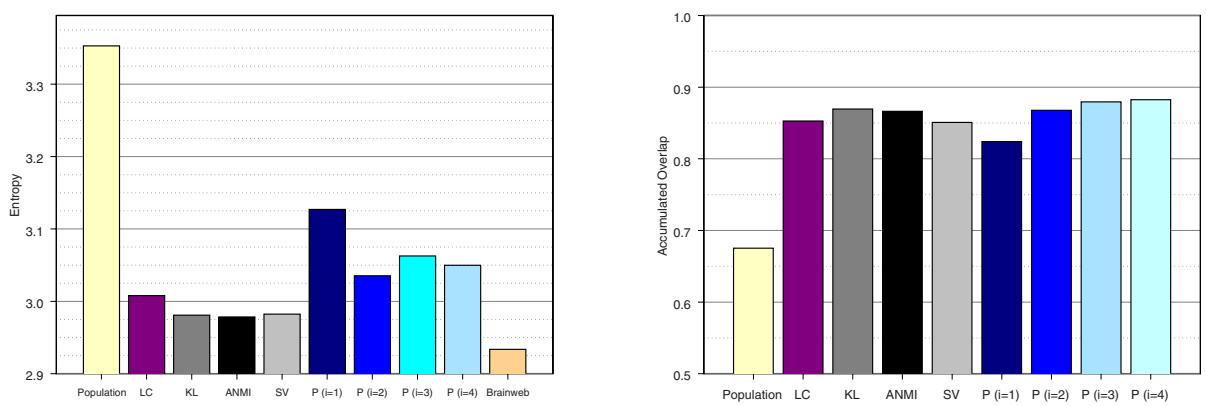

Fig. 3. Atlas entropies and accumulated overlaps showing consistency of registration 


\subsection{D MR Image Registration}

The best-performing intensity (ANMI, pairwise) and segmentation (KL) algorithms have also been tested on real 3D MR data for 12 subjects. The atlases are shown in Figure 4. These subjects have hard segmentations of 83 tissue classes obtained by expert manual segmentation, using an extension of an existing protocol [9]. The accumulated overlaps obtained are shown in Table 1 , showing similar performance of the metrics to the $2 \mathrm{D}$ case. It can be seen that while four iterations of the pairwise registration appears to give the best overlap, the overall deformation field is not zero. This means that the coordinate system of the final atlas does not truly represent the average of the population. Figure 4 shows that the total deformation in both groupwise cases is equal to zero, and the resulting atlases therefore describe the average shape of the population.

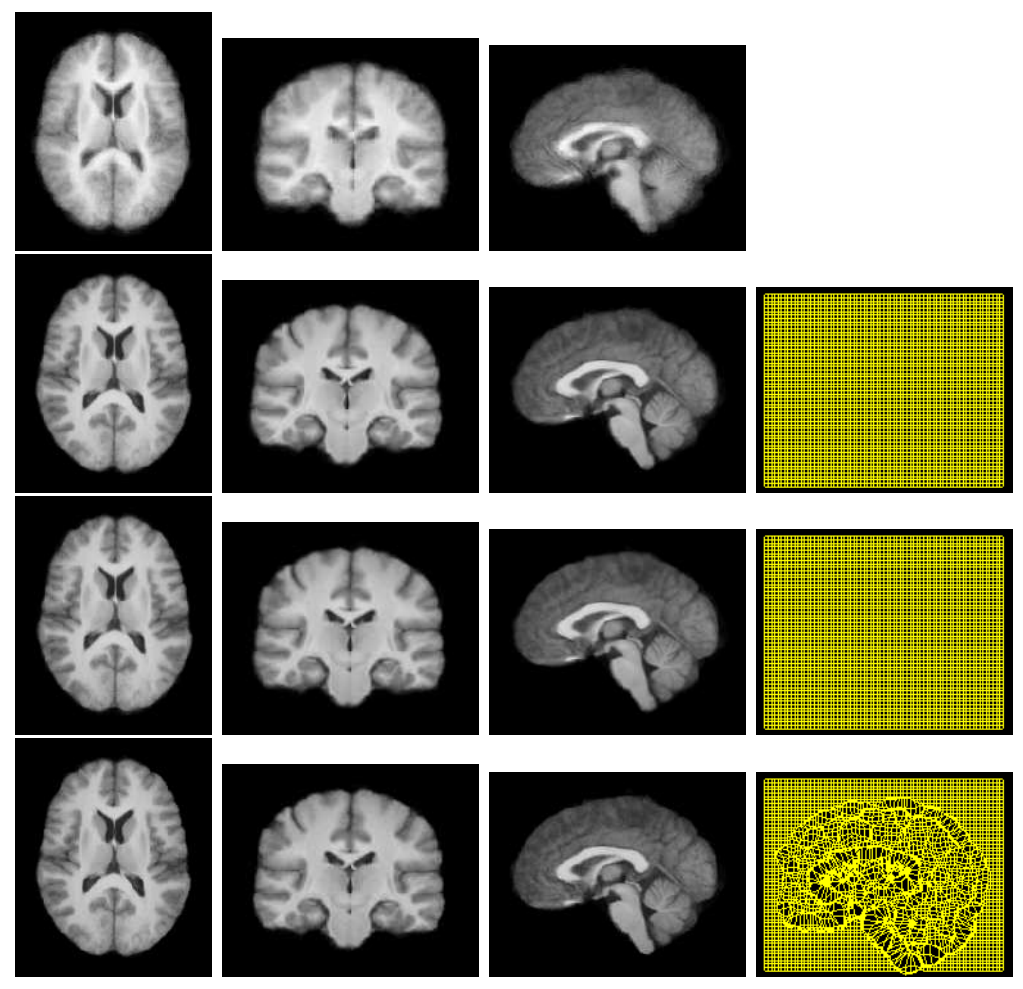

Fig. 4. Top row: axial, coronal and sagittal sections of affinely-aligned $123 \mathrm{D}$ adult subjects. Subsequent rows: groupwise registration using KL; groupwise registration using ANMI; fourth iteration of pairwise re-registration to average shape. Far right column: total deformation field (sagittal section) using each similarity metric. 
Table 1. Accumulated overlaps of 12 3D subjects registered to their average shape

\begin{tabular}{|c|c|c|c|c|}
\hline Affine & Pairwise (i=0) & Pairwise (i=4) & KL & ANMI \\
\hline \hline 0.56 & 0.60 & 0.63 & 0.62 & 0.61 \\
\hline
\end{tabular}

\section{Discussion}

This paper compares similarity measures for groupwise registration techniques. A novel similarity metric which extends the popular NMI metric to assess multisubject similarity has also been proposed. It was found that this metric, and the KL metric for when probabilistic segmentations are available, performed comparably to average atlas construction using four iterations of pairwise registration. Although this work does not constitute a full survey into the groupwise registration techniques available, it does demonstrate the relative performance of the methods considered, and provides an additional comparison with pairwise methods for atlas construction.

\section{References}

1. Studholme, C.: Simultaneous population based image alignment for template free spatial normalisation of brain anatomy. In: Gee, J.C., Maintz, J.B.A., Vannier, M.W. (eds.) WBIR 2003. LNCS, vol. 2717, pp. 81-90. Springer, Heidelberg (2003)

2. Joshi, S., Davis, B., Jomier, M., Gerig, G.: Unbiased diffeomorphic atlas construction for computational anatomy. NeuroImage 23, S151-S160 (2004)

3. Bhatia, K.K., et al.: Consistent groupwise non-rigid registration for atlas construction. In: ISBI 2004, pp. 908-911 (2004)

4. Twining, C., et al.: A a unified information-theoretic approach to groupwise nonrigid registration and model building. In: Christensen, G.E., Sonka, M. (eds.) IPMI 2005. LNCS, vol. 3565, pp. 1-14. Springer, Heidelberg (2005)

5. Zollei, L., et al.: Efficient population registration of 3D data. In: ICCV (2005)

6. Lorenzen, P., et al.: Multi-modal image set registration and atlas formation. Medical Image Analysis 10(3), 440-451 (2006)

7. Rueckert, D., et al.: Non-rigid registration using free-form deformations: Application to breast MR images. IEEE TMI 18(8), 712-721 (1999)

8. Marsland, S., et al.: Groupwise non-rigid registration using polyharmonic clampedplate splines. In: Ellis, R.E., Peters, T.M. (eds.) MICCAI 2003. LNCS, vol. 2878, pp. 771-779. Springer, Heidelberg (2003)

9. Hammers, A., et al.: Three-dimensional maximum probability atlas of the human brain, with particular reference to the temporal lobe. HBM 19, 224-247 (2003)

10. van Leemput, K., et al.: Automated model-based bias field correction of MR images of the brain. IEEE TMI 18(10), 885-896 (1999)

11. Kullback, S., Leibler, R.A.: On information and sufficiency. Annals of Mathematical Statistics 22(1), 79-86 (1951)

12. Guimond, A., et al.: Average brain models: A convergence study. Computer Vision and Image Understanding 77(9), 192-210 (2000) 
13. Rueckert, D., et al.: Automatic construction of 3-d statistical deformation models of the brain using nonrigid registration. IEEE TMI 22(8), 1014-1025 (2003)

14. Crum, W.R., et al.: Generalised overlap measures for assessment of pairwise and groupwise image registration and segmentation. In: Duncan, J.S., Gerig, G. (eds.) MICCAI 2005. LNCS, vol. 3749, pp. 99-106. Springer, Heidelberg (2005)

15. Rao, A., et al.: Spatial transformation of motion and deformation fields using nonrigid registration. IEEE TMI 23(9), 1065-1076 (2004) 